\title{
Diagnóstico y tratamiento temprano de neumonía ante la pandemia por COVID-19 en San Luis Potosí. ¿Es posible implementar una estrategia para lograrlo?
}

\author{
Early pneumonia diagnosis and treatment during COVID-19 \\ pandemic. Is there a feasible strategy to accomplish this?
}

\author{
Alejandro Gómez-Gómez,* María José Gómez-Escanamé,* Lidya Sánchez-Ramos, ${ }^{\ddagger}$ Daniel E Noyola* \\ * Facultad de Medicina. Universidad Autónoma de San Luis Potosí, México; \\ ${ }^{\ddagger}$ Hospital General ISSSTE San Luis Potosí, México.
}

\section{SITUACIÓN ACTUAL EN SAN LUIS POTOSÍ (SLP)}

Durante las últimas ocho semanas, en la ciudad de San Luis Potosí (SLP), México, se ha observado un incremento importante en el número de casos mayores de 25 años

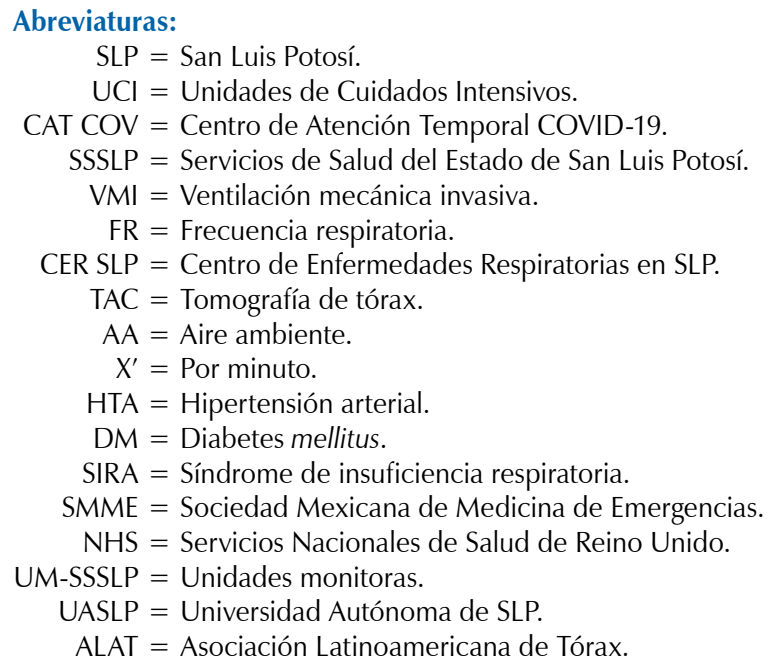

Citar como: Gómez-Gómez A, Gómez-Escanamé MJ, Sánchez-Ramos L, Noyola DE. Diagnóstico y tratamiento temprano de neumonía ante la pandemia por COVID-19 en San Luis Potosí. ¿Es posible implementar una estrategia para lograrlo? Neumol Cir Torax. 2020; 79 (4): 214-220. https:// dx.doi.org/10.35366/97962 hospitalizados con diagnóstico de neumonía, 55-64\% de ellos son reportados con neumonías graves o con necesidad de atención en unidades de cuidados intensivos (UCI). ${ }^{1} \mathrm{Si}$ el incremento en infecciones graves por SARS-CoV-2 continúa, puede llegar a presentarse una saturación del sistema de salud, por lo que convendría obtener un diagnóstico temprano de neumonía por COVID-19, reducir el número de ingresos hospitalarios y tratar a las personas en un centro de atención temporal para enfermos COVID-19 (CAT COV), o bien, en casa, iniciativa de los Servicios de Salud del Estado de San Luis Potosí (SSSLP). En SLP, el promedio de días de evolución desde el inicio de los primeros síntomas de COVID-19 al ingreso hospitalario es de nueve a 11 días (datos no publicados del Hospital General de Soledad de SSSLP), muy probablemente en fase inflamatoria de la enfermedad. ${ }^{2}$ La mortalidad descrita en pacientes críticos en diferentes países oscila entre 24 y $88 \% \cdot^{3-5}$

\section{¿DAÑO MECÁNICO AL PULMÓN POR POLIPNEA?}

Durante los últimos 25 años, se ha podido trasladar el conocimiento de investigación en animales a humanos en relación con la perpetuación de daño pulmonar en pacientes bajo ventilación mecánica invasiva (VMI), y que parece replicarse en pacientes no ventilados. Un incremento en la frecuencia respiratoria (FR) y profundidad de la inspiración (polipnea), con acentuación de las oscilaciones de presión intrapleural más marcada en las zonas dependientes en sujetos en decúbito supino (pendelluft), ha demostrado producir biotrauma y daño pulmonar autoinducido por el paciente (P-SILI, patient self-inflicted lung injury) a consecuencia de la liberación de citocinas a nivel pulmonar, producto de fuerzas 
de tensión generadas entre alvéolos sanos y enfermos, y en la interfase de vías aéreas de pulmón reclutado y pulmón colapsado por edema pulmonar y secreciones. ${ }^{6-10}$ Esto, traducido al terreno clínico cotidiano, puede observarse en casos de polipnea como compensación a la hipoxemia, y con frecuencia no percibida por el paciente. La hipoxemia silenciosa, bien descrita en sujetos con neumonía por COVID-19, puede ser producto de una respuesta inadecuada del centro respiratorio en ciertos enfermos de origen variado, o una interpretación errónea por parte del paciente; es posible que esto perpetúe o magnifique el daño pulmonar en etapas tempranas de neumonía por COVID-19.11,12

\section{FACTIBILIDAD DE TRATAMIENTO EN CASA}

En un Centro de Enfermedades Respiratorias en SLP (CER SLP), se trataron 68 casos con definición operacional de COVID-19 y diagnóstico temprano de neumonía por SARS-CoV-2 demostrada por rt-PCR y/o serología para SARS-CoV-2, con 6-8 días de inicio de síntomas; se utilizó un puntaje de NEWS2 (National Early Warning Score 2) de 4-6 basado principalmente en dos parámetros: $\mathrm{SaO}_{2}$ 9293\% al aire ambiente (AA) y FR 21-24 por minuto $\left(x^{\prime}\right)$, con polipnea (patrón respiratorio con uso de músculos torácicos superiores y esternocleidomastoideos en etapas tempranas), como criterios para solicitar TAC de tórax. El rango de edad fue entre 40 y 60 años, y las comorbilidades más frecuentes fueron hipertensión arterial (HTA) y diabetes mellitus (DM) controlada. El grado de afección por TAC fue moderado, $30-50 \%$ de afección total. El promedio de $\mathrm{SaO}_{2}$ fue de $92 \%$ AA y de FR de $23 x^{\prime}$ en la primera consulta; 38 tenían un puntaje de NEWS2 de 4 (verde: riesgo bajo) basado en $\mathrm{SaO}_{2}$ 92-93\% y FR $22-24 x^{\prime}$ con polipnea. Treinta y seis (56\%) no percibían disnea. El 84\% de los que ameritaban hospitalización fueron rechazados en servicios de urgencias y recibieron tratamiento en casa, $29 \%$ recibieron baricitinib durante 10 días para tratar síndrome hiperinflamatorio y deterioro respiratorio (disminución de $\mathrm{SaO}_{2}>5 \%$ del basal al aire ambiente); después de 48 horas de tratamiento con dexametasona, oxigenoterapia y decúbito prono, ninguno presentó infecciones sobreagregadas y sólo 11\% requirió hospitalización ulterior por deterioro, resuelto con oxigenoterapia de alto flujo, con promedio de seis días de estancia hospitalaria; ningún sujeto falleció o requirió VMI. Éstas son observaciones en grupos de pacientes con afección más temprana que los que se han estudiado de manera habitual, y es factible que se puedan encontrar intervenciones más eficaces que en enfermos con daño pulmonar grave.

\section{PREDICTORES DE GRAVEDAD Y DETERIORO}

En cohortes de casos con infección por SARS-CoV-2 analizadas en distintos países, se han descrito diversas carac- terísticas presentes con mayor frecuencia en aquéllos que progresan a síndrome de insuficiencia respiratoria aguda (SIRA) y necesidad de VMI, entre los que se encuentran: más de 10 días transcurridos entre los primeros síntomas de la infección y la admisión hospitalaria; presencia de disnea; sensación de pecho apretado; FR mayor a $22 x^{\prime}$ y $\mathrm{SaO}_{2} \mathrm{AA}$ en reposo igual o menor a $93 \% .^{13-18}$ Otros predictores de gravedad identificados han sido la presencia de comorbilidades, edad avanzada, así como ciertos parámetros de laboratorio e imagen. ${ }^{15,19,20}$

Las escalas qSOFA, CURB 65, CURB 65 extendido y SMART COP son útiles para identificar pacientes con neumonía que tienen una alta probabilidad de requerir atención en la UCl. ${ }^{21-24}$ La escala de Fine (PSI/PORT) requiere para su cálculo gasometría arterial, $F R>30 x^{\prime}$ y fue diseñada para predecir riesgo de mortalidad y necesidad de hospitalización. ${ }^{25}$ Ninguno de estos instrumentos está diseñado para diagnosticar neumonía en forma temprana.

La perspectiva para iniciar el estudio de un sujeto con sospecha de neumonía basada en escalas diseñadas para su utilización a nivel hospitalario se refleja en diversos algoritmos creados para la atención de pacientes con COVID-19, en los cuales se requieren criterios como $\mathrm{SaO}_{2} \mathrm{AA}$ menor de $90 \%$ y FR de 25 x' para iniciar un abordaje diagnóstico; estos algoritmos fueron diseñados para valorar si una persona que acude a la sala de urgencias es candidato a hospitalizarse en un escenario de alta demanda de atención. ${ }^{26-30}$

Aplicar estos criterios para diagnosticar neumonía en el contexto de la pandemia por COVID-19 implica aceptar que una proporción importante de casos requerirá de estancia en la $\mathrm{UCl}$ y $\mathrm{VMI}$ de acuerdo con los factores de riesgo mencionados anteriormente.

Por otro lado, se indicaría envío a su domicilio a los sujetos con neumonías en etapa más temprana, con FR de 22-24 x' con polipnea discreta y $\mathrm{SaO}_{2}$ de 92-93\% AA, lo que sin intervenciones adicionales podría incrementar la presencia de daño pulmonar a través de los mecanismos descritos de biotrauma y P-SILI, en especial si presentan un incremento en la profundidad de los esfuerzos inspiratorios; es de esperarse que la progresión de la afección respiratoria resulte en la necesidad de hospitalización, con mayor riesgo de requerir $\mathrm{VMI}$ e incluso fallecer.

\section{ESCALA NEWS2 Y COMORBILIDADES}

Las Guías de la Sociedad Mexicana de Medicina de Emergencias $(\mathrm{SMME})^{31}$ permiten establecer en forma más temprana el diagnóstico de neumonía a través de la utilización de la escala NEWS2, ${ }^{32}$ la cual está basada en signos vitales, fácil de aplicar en el primer nivel de atención médica y urgencias, diseñada bajo dos principios básicos: detección temprana de enfermos agudos y respuesta rápida ante la presencia de deterioro clínico para actuar en 
forma oportuna. Dichos elementos pueden ser de utilidad si se aplican a una estrategia de diagnóstico temprano de neumonía por COVID-19. Un puntaje de NEWS2 de 5-6 (amarillo o riesgo intermedio) indica que el sujeto en cuestión debe ser admitido al Servicio de Urgencias para descartar neumonía por COVID19. ${ }^{31}$ Sin embargo, limitarse a los casos que tienen de 5-6 puntos (o mayor) para considerar el diagnóstico de neumonía puede retrasarlo en una proporción importante de pacientes que cuentan con puntajes de 4 (oximetría 92-93\% y FR 21-24 x' con polipnea). Una estrategia descrita por los Servicios Nacionales de Salud de Reino Unido (NHS) establece un corte de oximetría de pulso igual o menor a 94\% para la evaluación en servicios de emergencias y descartar neumonía por COVID-19. ${ }^{33}$ También se recomienda en varias guías «regionalizar» y adaptar las escalas y algoritmos de decisiones de acuerdo con el entorno y realidad locales..$^{34,35}$ Entre los predictores de mayor relevancia para gravedad y mortalidad en neumonía por COVID-19 se encuentran las comorbilidades; hay diversos puntajes utilizados para evaluar su importancia en la decisión de hospitalizar a un paciente, el propuesto en esta estrategia se obtuvo en México, y parece adecuar en orden de importancia la edad y las patologías más frecuentes asociadas con gravedad de COVID-19 en nuestro país. ${ }^{36}$

\section{DIAGNÓSTICO TEMPRANO DE NEUMONÍA POR COVID-19}

Un enfoque que pretende diagnosticar en forma temprana la presencia de neumonía en el contexto de la pandemia por COVID-19 se beneficiaría de manera considerable de instrumentos sencillos y de fácil acceso; algunos de ellos son la oximetría de pulso en casa, la medición de FR, valoración de polipnea y patrón respiratorio por parte del médico de primer nivel por telemedicina o frente al paciente. La ciudad de SLP se encuentra a una altitud de 1,864 metros sobre el nivel del mar, por lo que la $\mathrm{SaO}_{2}$ normal esperada en personas sin enfermedades respiratorias crónicas es de $96 \%$ y la FR de 17 a $20 x^{\prime} .37,38$ Si se acepta una variabilidad de 2-3\% de algunos oxímetros de pulso, puede considerarse como límite bajo normal una $\mathrm{SaO}_{2}$ de $93 \%$, así como una FR de $20 x^{\prime}$ como el límite superior normal en esta ciudad. ${ }^{39,40}$

En la Tabla 1 se muestra una propuesta que comienza a implementarse en el CAT COV y unidades monitoras (UM-SSSLP) de primer nivel de los SSSLP en conjunto con la Facultad de Medicina de la Universidad Autónoma de San Luis Potosí (UASLP). Un protocolo similar y más estructurado es utilizado desde hace varios meses en la Unidad Temporal COVID-19 (UTC-19) en la Ciudad de México, con excelentes resultados. ${ }^{41}$ Los objetivos son diagnosticar

Tabla 1: Evaluación y referencia de pacientes sintomáticos que acuden a consulta de primer nivel de atención por COVID-19 en SLP.

\begin{tabular}{|c|c|c|}
\hline \multicolumn{3}{|c|}{ Unidades monitoras y kioscos: formato electrónico estandarizado (síntomas COVID-19, FR > 30 x', edad, NEWS2 con polipnea) } \\
\hline Hogar & CAT COV & $\begin{array}{c}\text { NEUMONÍA ESTABLECIDA } \\
\text { HOSPITALIZACIÓN }\end{array}$ \\
\hline $\begin{array}{l}\text { NEWS2 } 4 \text { puntos: } \mathrm{SaO}_{2} 92-93 \% \text { y FR } 21-24 \mathrm{x}^{\prime} \\
\text { con polipnea, y riesgo bajo de comorbilidades }{ }^{\ddagger} \\
\text { y< } 65 \text { años } \\
\text { (Visita domiciliaria diaria por brigadas. Diario de } \\
\text { signos y síntomas en celular, telemedicina) }\end{array}$ & $\begin{array}{l}\text { - NEWS2 } 4 \text { puntos: } \mathrm{SaO}_{2} \text { 92-93\% y FR } 21-24 \text { x' con } \\
\text { polipnea, y riesgo intermedio de comorbilidades }{ }^{\ddagger} 0 \\
\text { edad > } 65 \text { años } \\
\text { - NEWS2 5-6 puntos y riesgo bajo o intermedio de } \\
\text { comorbilidades } \\
\text { (Apoyo infectología y neumología por telemedicina. } \\
\text { No hay UCl) }\end{array}$ & $\begin{array}{l}\text { - } \quad \text { FR igual } 0>30 x^{\prime} \\
\text { - NEWS2 }>6 \text { puntos } \\
\text { - NEWS2 5-6 puntos } \\
\text { conriesgo elevado de } \\
\text { comorbilidades }{ }^{\ddagger}\end{array}$ \\
\hline \multicolumn{2}{|c|}{$\begin{array}{l}\text { Tratamiento estandarizado en SLP: a) oxigenoterapia en caso de } \mathrm{SaO}_{2} \text { igual o menor a } 92 \% \text { y a flujo necesario } \\
\text { para lograr } \mathrm{SaO}_{2}>92 \% \text { y decúbito prono } 4 \text { horas por turno; b) azitromicina } 500 \mathrm{mg} \text { al día; c) dexametasona } 6 \\
\text { mg diarios por } 10 \text { días, si hay neumonía comprobada y } \mathrm{SaO}_{2}<92 \% \S \text { (otro esteroide si dexametasona no dispo- } \\
\text { nible); d) anticoagulación profiláctica y dosis ajustada de acuerdo con dímero } \mathrm{D} \text {; e) valorar uso de cefalosporina } \\
\text { de tercera generación; f) en caso de falla a tratamiento con dexametasona" valorar baricitinib } 2 \text { a } 4 \text { mg cada } 12 \\
\text { horas por } 10 \text { días, previo consentimiento del paciente }\end{array}$} & \\
\hline
\end{tabular}

Se presenta una propuesta estandarizada, sencilla y accesible, dirigida al médico de primer nivel, que le permita identificar a pacientes que requieran estudios para descartar neumonía por COVID-19 y sugiere el sitio adecuado de referencia para su tratamiento basado en 4 puntos: puntaje NEWS2, edad, puntaje de comorbilidades y FR inicial de $30 x^{\prime}$.

* NEWS2 mínimo de 4 puntos (bajo-intermedio) basado en $\mathrm{SaO}_{2}$ 92-93\% y FR 21-24 x' con polipnea detectada, o NEWS2 5-6 puntos o mayor: radiografía de tórax, BH, QS, PCR, dímero D, ferritina, DHL, CPK, troponina, PFH.

${ }^{\ddagger}$ Riesgo de comorbilidades basado en puntaje de referencia bibliográfica 36 (Bello-Chavolla OY et al).

$\S$ Basado en referencias 45 y 46.

" Falla a tratamiento con dexametasona se basa en dos parámetros: a) deterioro respiratorio en paciente con neumonía demostrada, reducción de $\mathrm{SaO}_{2}>5 \%$ al aire ambiente después de 48-72 horas de tratamiento, aumento de requerimiento de flujo oxígeno, y b) hiperinflamación persistente: incremento de PCR a valores $>75 \mathrm{mg} / \mathrm{L}$, ferritina > 1,000 (propuesta destinada para su discusión en el estado de San Luis Potosí). 


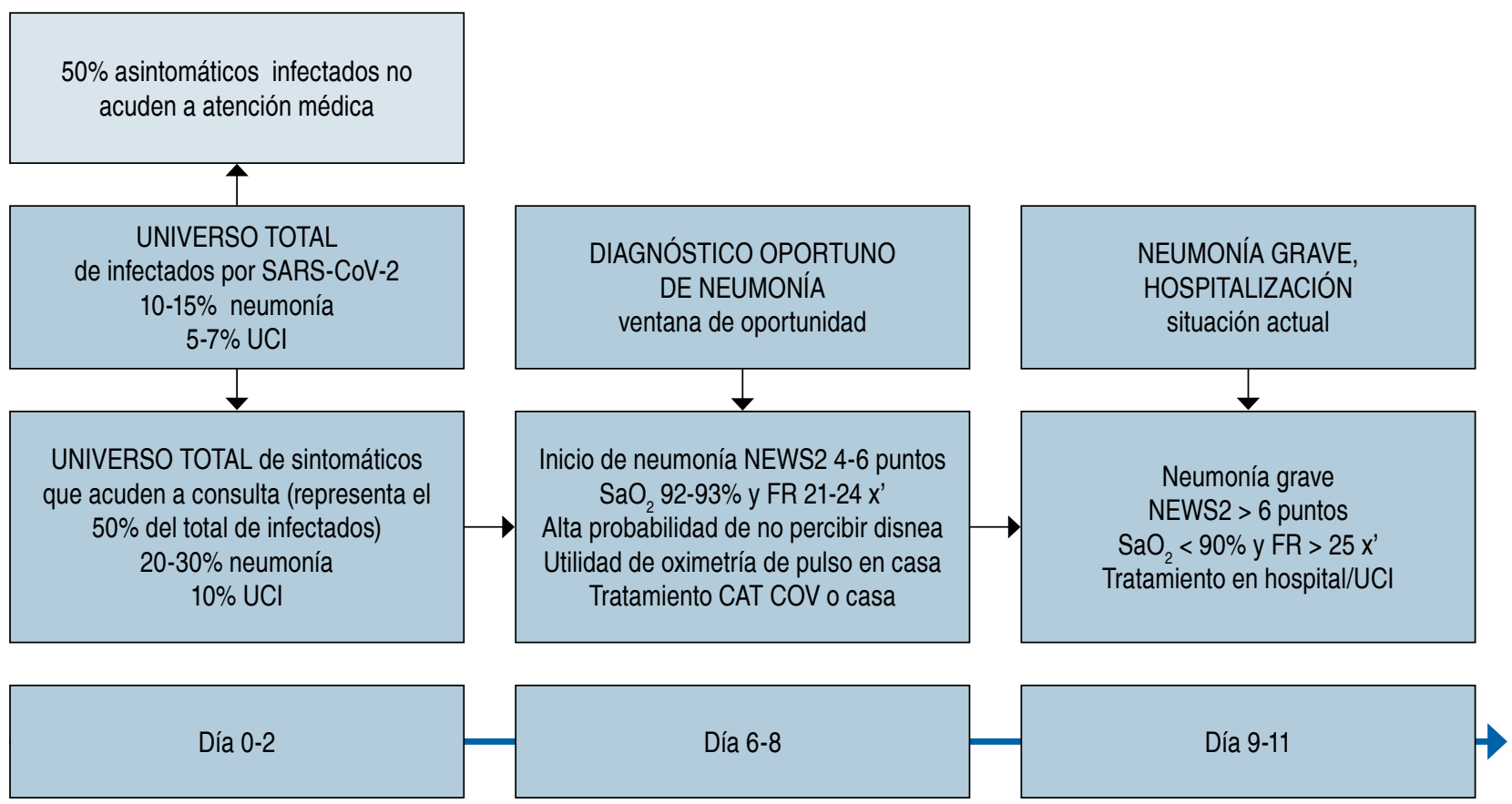

Figura 1: En el extremo izquierdo se muestra el universo de casos que acuden a consulta; en realidad representan sólo el $50 \%$ de los infectados, por lo que la posibilidad de enfrentarse a enfermos con neumonía en quienes acuden a consulta por síntomas de COVID-19 es de 20-30\%. En el extremo derecho se representan los casos atendidos por neumonía que ingresan a los 9-11 días de evolución, con mayor riesgo de presentaciones graves y necesidad de la UCI. En la parte central, se presenta la ventana de oportunidad de diagnosticar y tratar en forma temprana a los pacientes con neumonía por COVID-19, lo que puede reducir el número de hospitalizaciones.

neumonía por COVID-19 en forma temprana, ofrecer tratamiento y seguimiento estandarizados en forma oportuna y ayudar a reducir el número de casos que requieran internarse en hospitales y unidades de la UCl (Figura 1). Es importante señalar que esta estrategia no contempla sujetos con patologías pulmonares crónicas con $\mathrm{SaO}_{2}$ bajas en forma crónica, para este grupo de enfermos podría recomendarse una disminución $>3-4 \%$ o mayor dado el comportamiento de la curva de disociación de la hemoglobina a $\mathrm{SaO}_{2}<90 \%$ de su valor basal, acompañado de incremento de disnea y síntomas respiratorios, o utilizar el puntaje de NEWS2 para $\mathrm{SaO}_{2}$ en pacientes con hipoxemia crónica. ${ }^{32}$ La detección temprana puede extenderse a casa con el uso de oxímetros de pulso, que también registran FR y conexión vía Bluetooth a teléfonos celulares. ${ }^{42}$

La estrategia se basa en acciones a diferentes niveles: a) UM-SSSLP y kioscos distribuidos en diversos puntos de la ciudad, se invita a pacientes con síntomas respiratorios agudos, se toman signos vitales y aplican en forma estandarizada y protocolizada un cuestionario de síntomas de COVID-19 y escala NEWS2 con énfasis en detectar $\mathrm{SaO}_{2}$ y polipnea. Con un cálculo en tabletas electrónicas o celulares, se establece el riesgo de padecer neumonía, la necesidad de realizar estudios de laboratorio y radiografía de tórax, además del sitio potencial a donde debe referirse, ya sea a casa, CAT COV u hospital (Tabla 1); b) capacitación de médicos de primer nivel (de sistemas de salud públicos y privados) que les permita utilizar la oximetría de pulso en forma adecuada, reconocer un patrón respiratorio anormal en etapa incipiente y aplicar en forma estandarizada y protocolizada escalas y algoritmos de decisión; c) diseminación de información a la ciudadanía con mensajes simples: «alíviate si acudes a tiempo», «no esperes a que te falte el aire para buscar atención médica», «si tienes gripa, fiebre o tos, usa tu oxímetro de pulso en casa, puede salvar tu vida», etcétera.

\section{OXIGENOTERAPIA, DECÚBITO PRONO EN NO INTUBADOS Y OTROS TRATAMIENTOS}

Desde el punto de vista terapéutico, la oxigenoterapia y el decúbito prono pueden ser tratamientos útiles en etapas tempranas de neumonía por COVID-19 con $\mathrm{SaO}_{2}$ igual o menor a $92-93 \%$ y polipnea en $\mathrm{SLP}^{42-44}$ en conjunto con otros tratamientos recomendados por diversos organismos. ${ }^{26,27,45}$ El nivel de $\mathrm{SaO}_{2}$ recomendado para el inicio de oxigenoterapia es variado y en ocasiones responde a la opinión de los expertos. El consenso sobre el manejo de insuficiencia respiratoria aguda de Italia define hipoxemia como una $\mathrm{SaO}_{2}<94 \% .{ }^{35}$ Las Guías de la Asociación Lati- 
noamericana de Tórax (ALAT) sugieren iniciar oxigenoterapia con una $\mathrm{SaO}_{2}$ igual o menor a $94 \%$ y una $\mathrm{FR}>20$ x', y considerar el uso de decúbito prono. ${ }^{36}$

El uso de esteroides en COVID-19 ha sido demostrado en el estudio RECOVERY, en el cual se estableció su utilidad en casos que requirieron oxigenoterapia con y sin $\mathrm{VMI}$. Al revisar el protocolo y publicación de dicho estudio, no se define el nivel de $\mathrm{SaO}_{2}$ a partir del cual se utilizó oxigenoterapia y se dejó a libertad de cada hospital establecerlo. En el protocolo de dicho estudio, se menciona una $\mathrm{SaO} 2$ igual o menor a $92 \%$ y la presencia de proteína $\mathrm{C}$ reactiva a $75 \mathrm{mg} / \mathrm{dL}$ como criterio de estado hiperinflamatorio y de inclusión para la aplicación de tocilizumab versus terapia estándar. ${ }^{46,47}$ En el protocolo del estudio COVACTA, los criterios de inclusión para ser candidato a recibir tocilizumab fueron la presencia de neumonía confirmada por COVID-19 y una $\mathrm{SaO}_{2}$ igual o menor a $93 \%{ }^{48}$ Otra alternativa terapéutica evaluada en diversos estudios son los inhibidores de la vía JAK-STAT; baricitinib muestra un efecto antiviral al reducir la endocitosis y modula la respuesta inflamatoria a través de la inhibición de la cinasa JAK1/JAK2, y reducción de la fosforilación de STAT3 inducida por IL- $6 .{ }^{45}$ En el protocolo del estudio ACTT-2, los criterios de inclusión fueron sujetos hospitalizados con neumonía comprobada por COVID-19, $\mathrm{SaO}_{2}$ igual o menor a $94 \%$ o requerimiento de oxígeno suplementario (que se deja a criterio libre de definición por cada centro que participó en el estudio). ${ }^{49}$ Otro elemento de la presente propuesta es la utilización de tratamiento antiinflamatorio una vez realizado el diagnóstico temprano de neumonía por COVID-19. Con base en el efecto demostrado en el estudio RECOVERY en casos que requirieron oxigenoterapia, se plantea la posibilidad de utilizar esteroides en quienes presentan neumonía demostrada por radiografía o TAC de tórax, con niveles de $\mathrm{SaO}_{2}$ iguales o menores a 92\%; además, se plantea la posibilidad de añadir baricitinib en caso de deterioro respiratorio después de 48 horas de terapia con esteroides (reducción de $\mathrm{SaO}_{2}>5 \%$ al aire ambiente) o incremento de flujo de oxígeno e incremento de FR a más de 25 x'. Esta propuesta se basa en que el nivel de $\mathrm{SaO}_{2}$ que se recomienda para utilizar esteroides depende de la decisión de iniciar oxigenoterapia, la cual es libre para cada hospital, pero sigue la lógica de una reducción de 3 a $4 \%$ de la $\mathrm{SaO}_{2}$ normal para cada ciudad; en el caso de baricitinib, dado que el beneficio demostrado es en combinación con remdesivir, el cual no está disponible en México, se pretende que esta propuesta sea discutida, y eventualmente aceptada a nivel estatal para poder utilizarla hasta tener estudios con mayor número de casos que establezcan su beneficio en neumonía temprana por COVID-19. Asimismo, la utilización de este tratamiento requiere de autorización previa de la persona, con información detallada sobre los beneficios y riesgos potenciales.
Dada la situación actual de la pandemia por COVID-19, es importante generar estrategias que simplifiquen la identificación de sujetos con riesgo de desarrollar formas graves de la infección y eviten la sobrecarga a los hospitales. Esta propuesta requiere de validación en una cohorte con mayor número de casos, multicéntrico, idealmente aleatorizado y doble ciego, que tenga un número adecuado de personas con diagnóstico «temprano» (seis a ocho días) de neumonía demostrada por imagen.

En resumen, proponemos la identificación de casos con neumonía en fase temprana y la prescripción de oxigenoterapia, decúbito prono en pacientes despiertos, así como el uso de esteroides en presencia de hipoxemia $\left(\mathrm{SaO}_{2}\right.$ igual o menor a 92\%), y otras terapéuticas para síndrome hiperinflamatorio (tormenta de citocinas), los cuales estaban contraindicados o no se encontraban disponibles durante la pandemia de influenza $\mathrm{A}(\mathrm{H} 1 \mathrm{~N} 1)$ en el 2009. La implementación de estrategias adicionales sería de igual importancia, como búsqueda intencionada de súper propagadores y personas infectadas que representan un alto riesgo de transmisión (por ejemplo, trabajadores que ofrecen diversos servicios a domicilio, familias numerosas en condiciones de hacinamiento) para reducir el número de ingresos hospitalarios por neumonía.

\section{Agradecimientos}

A Servicios de Salud del Estado de San Luis Potosí y Facultad de Medicina de la UASLP por su apoyo. Dr. Rafael Valdés y personal de salud de UTC-19 por asesoría y capacitación de personal médico y de enfermería. A la Fundación Carlos Slim por su apoyo con plataformas electrónicas y de comunicación. Organismos de la sociedad civil Asociación Civil Chat Social, Grupo CO-Labora Sociedad Civil, que permiten permear esta información a la población y gestionar donaciones de recursos. Un reconocimiento y agradecimiento especial a todos los médicos y personal de salud que están al frente de unidades COVID-19 en hospitales de sistemas de salud públicos y privados, por su entrega y calidad.

\section{REFERENCIAS}

1. Comité Estatal para la Seguridad en Salud. Reportes del 1 al 20 de agosto de 2020. Gobierno del Estado de San Luis Potosí.

2. St. John AL, Rathore APS. Early insights into immune responses during COVID-19. J Immunol. 2020;205(3):555-564. Available in: https://doi.org/10.4049/jimmunol.2000526

3. Guan WJ, Ni ZY, Hu Y, Liang WH, Ou CQ, He JX, et al. Clinical characteristics of coronavirus disease 2019 in China. N Engl J Med. 2020;382(18):1708-1720. Available in: https://doi.org/10.1056/ nejmoa2002032

4. Quah P, Li A, Phua J. Mortality rates of patients with COVID-19 in the intensive care unit: a systematic review of the emerging literature. Crit Care. 2020;24(1):285. Available in: https://doi.org/10.1186/s13054-020-03006-1 
5. Richardson S, Hirsch JS, Narasimhan M, Crawford JM, McGuinn T, Davidson KW, et al. Presenting characteristics, comorbidities, and outcomes among 5700 patients hospitalized with Covid-19 in the New York City area. JAMA. 2020;323(20):2052-2059. Available in: https:// doi.org/10.1001/jama.2020.6775

6. Hubmayr RD, Kallet RH. Understanding pulmonary stress-restrain relationships in severe ARDS and its implications for designing a safer approach to setting the ventilator. Respir Care. 2018;63(2):219-226. Available in: https://doi.org/10.4187/respcare.05900

7. Brochard L, Slutsky A, Pesenti A. Mechanical ventilation to minimize progression of lung injury in acute respiratory failure. Am J Respir Crit Care Med. 2017;195(4):438-442. Available in: https://doi.org/10.1164/ rccm.201605-1081cp

8. Yoshida T, Grieco DL, Brochard L, Fujino Y. Patient self-inflicted lung injury and positive end-expiratory pressure for safe spontaneous breathing. Curr Opin Crit Care. 2020;26(1):59-65. Available in: https:// doi.org/10.1097/mcc.0000000000000691

9. Grieco DL, Menga LS, Eleuteri D, Antonelli M. Patient self-inflicted lung injury: implications for acute hypoxemic respiratory failure and ARDS patients on non-invasive support. Minerva Anestesiol. 2019;85(9):10141023. Available in: https://doi.org/10.23736/s0375-9393.19.13418-9

10. Madahar P, Beitler JR. Emerging concepts in ventilation-induced lung injury. F1000Res. 2020;9:F1000 Faculty Rev-222. Available in: https://doi.org/10.12688/f1000research.20576.1

11. Tobin MJ, Laghi F, Jubran A. Why COVID-19 silent hypoxemia is baffling to physicians. Am J Respir Crit Care Med. 2020;202(3):356360. Available in: https://doi.org/10.1164/rccm.202006-2157cp

12. Pérez-Padilla JR, Thirión-Romero II, Aguirre-Pérez T, RodríguezLlamazares S. ¿Qué tan silenciosa es la hipoxemia en COVID-19? Neumol Cir Torax. 2020;79(2):69-70. Disponible en: https://dx.doi. org/10.35366/94629

13. Wu C, Chen X, Cai Y, Xia J, Zhou X, Xu S, et al. Risk factors associated with acute respiratory distress syndrome and death in patients with coronavirus disease 2019 pneumonia in Wuhan, China. JAMA Intern Med. 2020;180(7):934-943. Available in: https://doi.org/10.1001/ jamainternmed.2020.0994

14. Li X, Xu S, Yu M, Wang K, Tao Y, Zhou Y, et al. Risk factors for severity and mortality in adult COVID-19 inpatients in Wuhan. J Allergy Clin Immunol. 2020;146(1):110-118. Available in: https://doi.org/10.1016/j. jaci.2020.04.006

15. Suleyman G, Fadel RA, Malette KM, Hammond C, Adbulla H, Entz $A$, et al. Clinical characteristics and morbidity associated with coronavirus disease 2019 in a series of patients in metropolitan Detroit. JAMA Netw Open. 2020;3(6):e20122270. Available in: https://doi. org/10.1001/jamanetworkopen.2020.12270

16. Zhang J, Wang X, Jia X, Li J, Hu K, Chen G, et al. Risk factors for disease severity, unimprovement, and mortality in COVID-19 patients in Wuhan, China. Clin Microb Infect. 2020;26(6):767-772. Available in: https://doi.org/10.1016/j.cmi.2020.04.012

17. Sun Q, Qiu H, Huang M, Yang Y. Lower mortality of COVID-19 by early recognition and intervention: experience from Jiangsu Province. Ann Intensive Care. 2020;10(1):33. Available in: https://doi.org/10.1186/ s13613-020-00650-2

18. Ihle-Hansen H, Berge T, Tveita A, Rønning EJ, Ernø PE, Andersen EL, et al. Covid-19: symptoms, course of illness and use of clinical scoring systems for the first 42 patients admitted to a Norwegian local hospital. Tidsskr Nor Laegeforen. 2020;140(7). Available in: https:// doi.org/10.4045/tidsskr.20.0301
19. Ciceri F, Castagna A, Rovere-Querini P, De Cobelli F, Ruggeri A, Galli $\mathrm{L}$, et al. Early predictors of clinical outocomes of COVID-19 outbreak in Milan Italy. Clin Immunol. 2020;217: 108509. Available in: https:// doi.org/10.1016/j.clim.2020.108509

20. Tian W, Jiang W, Yao J, Nicholson CJ, Li RH, Sigurslid HH, et al. Predictors of mortality in hospitalized COVID-19 patients: a systematic review and meta-analysis. J Med Virol. 2020;10.1002/jmv.26050. Available in: https://doi.org/10.1002/jmv.26050

21. Seymour CW, Liu VX, Iwashyna TJ, Brunkhorst FM, Rea TD, Scherag $A$, et al. Assessment of clinical criteria for sepsis: for the third international consensus definitions for sepsis and septic shock (Sepsis-3). JAMA. 2016;315(8):762-774. Available in: https://doi.org/10.1001/ jama.2016.0288

22. Lim WS, van der eerden MM, Laing R, Boersma WG, Karalus N, Town $\mathrm{Gl}$, et al. Defining community acquired pneumonia severity on presentation to hospital: an international derivation and validation study. Thorax. 2003;58(5):377-382. Available in: https://doi.org/10.1136/ thorax.58.5.377

23. Liu JL, Xu F, Zhou H, Wu XJ, Shi LX, Lu RQ, et al. Expanded CURB65: a new score system predicts severity of community-acquired pneumonia with superior efficiency. Sci Rep. 2016;6:22911. Available in: https://doi.org/10.1038/srep22911

24. Charles PGP, Wolfe R, Whitby M, Fine MJ, Fuller AJ, Stirling R, et al. SMART-COP: a tool for predicting the need for intensive respiratory or vasopressor support in community-acquired pneumonia. Clin Infect Dis. 2008;47(3):375-384. Available in: https://doi.org/10.1086/589754

25. Fine MJ, Auble TE, Yealy DM, Hanusa BH, Weissfeld LA, Singer DE, et al. A prediction rule to identify low-risk patients with communityacquired pneumonia. N Engl J Med. 1997;336(4):243-250. Available in: https://doi.org/10.1056/nejm199701233360402

26. Gobierno de México. Flujograma de atención médica para COVID-19 en el primer nivel de atención. [Fecha de consulta: 16 de agosto de 2020] Disponible en: https://coronavirus.gob.mx/wp-content/ uploads/2020/04/Flujograma_Atencion_Primer_Nivel_13042020.pdf

27. Gobierno de México, IMSS. Algoritmos interinos para la atención del COVID-19. 31 julio 2020. [Fecha de consulta: 16 de agosto de 2020] Disponible en: http://educacionensalud.imss.gob.mx/es/system/files/ Algoritmos_interinos_COVID19_CTEC.pdf.

28. Elizalde González JJ, Fortuna Custodio JA, Luviano García JA, Mendoza Romero VM, Mijangos Méndez JC, Olivares Durán EM, et al. Guía COVID-19 para la atención del paciente crítico con infección por SARS-CoV-2 Colegio Mexicano de Medicina Crítica. Med Crit. 2020;34(1):7-42. Disponible en: https://dx.doi. org/10.35366/93279

29. Siordia Jr JA. Epidemiology and clinical features of COVID-19: a review of current literature. J Clin Virol. 2020;127:104357. Available in: https://doi.org/10.1016/j.jcv.2020.104357

30. BMJ Best Practice. Enfermedad de coronavirus 2019 (COVID-19). Agosto 10, 2020. [Fecha de consulta: 18 de agosto de 2020] Disponible en: https://bestpractice.bmj.com/topics/es-es/3000168.

31. Romero-Hernández S, Saavedra Uribe J, Zamarrón López El, Pérez-Nieto OR, Figueroa Uribe AF, Guerrero Gutiérrez MA, et al. Protocolo de atención para COVID-19 (SARS-CoV-2) de la Sociedad Mexicana de Medicina de Emergencias. 2020. Available in: https:// doi.org/10.13140/RG.2.2.16460.97922

32. Royal College of Physicians. National Early Warning Score (NEWS) 2: Standardizing the assessment of acute-illness severity in the NHS. Updated report of a working party. London: RCP; 2017. 
33. UK Nacional Health Service. NHS guidance for pulse oximetry to detect early deterioration of patients with COVID-19 in primary and community care setting. [Access date: 2020 August 16] Available in: https://www.england.nhs.uk/coronavirus/publication/pulse-oximetryto-detect-early-deterioration-of-patients-with-covid-19-in-primaryand-community-care-settings/.

34. Vitacca M, Nava S, Santus P, Harari S. Early consensus management for non-ICU acute respiratory failure SARS-CoV-2 emergency in Italy: from ward to trenches. Eur Respir J. 2020;55(5):2000632. Available in: https://doi.org/10.1183/13993003.00632-2020

35. Vega ML, Sirotti C, Montiel G, Sandoval GJL, Baz EM, Cohen TM, et al. AAMR/Departamentos Medicina Crítica/Sueño ALAT. Recomendaciones para el manejo invasivo y no invasivo de la insuficiencia respiratoria hipoxémica por COVID-19. Respirar. 2020;12(1): Separata (3)1-30.

36. Bello-Chavolla OY, Bahena-López JP, Antonio-Villa NE, VargasVázquez A, González-Díaz A, Márquez-Salinas A, et al. Predicting mortality due to SARS-CoV-2: a mechanistic score relating obesity and diabetes to COVID-19 outcomes in Mexico. J Clin Endocrinol Metab. 2020;105(8):dgaa346. Available in: https://doi.org/10.1210/clinem/dgaa346

37. Ministerio de Salud Colombia, OMS, OPS. Uso e interpretación de la oximetría de pulso. Convenio 519 de 2015. Bogotá. D.C.: 2016. [Fecha de consulta: 20 de agosto de 2020] Disponible en: https:// www.minsalud.gov.co/sites/rid/Lists/BibliotecaDigital/RIDE/VS/PP/ ENT/uso-interprtn-oximetria-pulso.pdf

38. Vázquez García JC, Pérez-Padilla R. Valores gasométricos estimados para las principales poblaciones y sitios a mayor altitud en México. Rev Inst Nal Enf Resp Mex. 2000;13(1):6-13.

39. Hess DR. Pulse oximetry: Beyond $\mathrm{SpO}_{2}$. Respir Care. 2016;61(12):16711680. Available in: https://doi.org/10.4187/respcare.05208

40. Organización Panamericana de la Salud. Aspectos técnicos y regulatorios sobre el uso de oxímetros de pulso en el monitoreo de pacientes con COVID-19. 7 agosto 2020. [Fecha de consulta: 22 de agosto de 2020] Disponible en: https://iris.paho.org/bitstream/handle/10665.2/52551/OPSHSSMTCOVID-19200029_spa. pdf?sequence $=1$ \&isAllowed $=y$.
41. Manual de Operaciones y atención clínica. Fuente: datos de la Unidad Temporal COVID-19, UTC-19 Citibanamex.

42. Quaresima V, Ferrari M. More in pulse oximetry for monitoring patients with COVID-19 at home. Ann Am Thorac Soc. 2020. Available in: https://doi.org/10.1513/annalsats.202006-701le

43. Koeckerling D, Barker J, Mudalige NL, Oyefeso O, Pan D, Pareek M, et al. Awake prone positioning in COVID-19. Thorax. 2020;75(10):833834. doi: 10.1136/thoraxjnl-2020-215133.

44. Thompson AE, Ranard BL, Wei Y, Jelic S. Prone positioning in awake, nonintubated patients with COVID-19 hypoxemic respiratory failure. JAMA Intern Med. 2020:e203030. Available in: https://doi.org/10.1001/ jamainternmed.2020.3030

45. COVID-19 Treatment Guidelines Panel. Coronavirus Disease 2019 (COVID-19) Treatment Guidelines. National Institutes of Health. [Access date: 2020 September 25] Available in: https://files.covid19treatmentguidelines.nih.gov/guidelines/covid19treatmentguidelines.pdf.

46. RECOVERY Collaborative group, Horby P, Lim WS, Emberson JR, Mafham M, Bell JL, Linsell L, et al. Dexamethasone in hospitalized patients with Covid-19-Preliminary report. N Engl J Med. 2020;NEJMoa2021426. Available in: https://doi.org/10.1056/nejmoa2021436

47. Randomised evaluation of COVID-19 therapy (RECOVERY). [Access date: 2020 September 20] Available in: https://www.recoverytrial.net/ files/recovery-protocol-v7-0-2020-06-18.pdf.

48. A study to evaluate the safety and efficacy of tocilizumab in patients with severe COVID-19 pneumonia (COVACTA). [Access date: 2020 September 20] Available in: https://clinicaltrials.gov/ct2/show/ NCT04320615.

49. Adaptive COVID-19 treatment trial 2 (ACTT-2). [Access date: 2020 September 18] Available in: https://clinicaltrials.gov/ct2/show/ NCT04401579.

Conflicto de intereses: Los autores declaran no tener conflicto de intereses. 